\title{
The Occurrence of CMV-Specific Short RNAs in Transgenic Tobacco Expressing Virus-Derived Double- Stranded RNA is Indicative of Resistance to the Virus
}

\author{
Kriton Kalantidis, ${ }^{1}$ Stavros Psaradakis, ${ }^{1}$ Martin Tabler, ${ }^{1}$ and Mina Tsagris ${ }^{1,2}$ \\ ${ }^{1}$ Foundation for Research and Technology-Hellas Institute of Molecular Biology and Biotechnology, P.O. Box 1527, 71110 \\ Heraklion, Crete, Greece; ${ }^{2}$ Biology Department, University of Crete, 71110 Heraklion, Crete, Greece.
}

Submitted 26 November 2001. Accepted 17 April 2002.

\begin{abstract}
Expression or introduction of double-stranded (ds)RNA in eukaryotic cells can trigger sequence-specific gene silencing of transgenes, endogenes, and viruses. Transgenic plants producing dsRNAs with homology to viral sequences are likely to exhibit pathogen-derived resistance to the virus. Cucumber mosaic virus (CMV), a very widespread virus with over 1,000 host species, has the natural ability to suppress silencing in order to establish infection. Here, we report the generation of transgenic tobacco lines, where a DNA transgene containing an inverted repeat of CMV cDNA had been introduced. Expression of this DNA construct delivered an RNA transcript that is able to form an intramolecular double strand. Transgenic plants were challenged with CMV. Three categories of plants could be discriminated: susceptible plants, which typically reacted with milder symptoms than the wild-type control; a "recovery" phenotype, in which newly emerging leaves were free of symptoms; and plants that showed complete resistance. Northern analysis showed that the expression of CMV dsRNA caused, in some transgenic lines, the generation of short RNAs characteristic of posttranscriptional gene silencing. Those lines were CMV resistant. The correlation between the detection of short RNAs and virus resistance provides a molecular marker that makes it possible to predict success in attempts to engineer virus resistance by dsRNA.
\end{abstract}

Several years ago, Dougherty and colleagues observed that the expression of a part of an untranslatable viral gene might confer resistance to that virus (Lindbo and Dougherty 1992; Lindbo et al. 2001). They concluded further that the resistance was based on a degradation system localized in the cytosol, indicative of regulation at posttranscriptional level (Dougherty et al. 1994). These characteristics were surprisingly similar to the phenomenon of "cosuppression", a situation observed when heterologous transgenes were expressed from (usually strong) promoters: Very often, the transgene was posttranscriptionally silenced. If an endogene had a sufficient degree of sequence similarity it was often silenced, too (Kooter et al. 1999; Matzke and Matzke 1995; Napoli et al. 1990). Since then, posttranscriptional gene silencing (PTGS) has been found in many eukaryotic organisms, fungi, invertebrates, vertebrates,

Corresponding author: M. Tsagris, E-mail: tsagris@imbb.forth.gr

* The $\boldsymbol{e}$-Xtra logo stands for "electronic extra" and indicates Figure 2 is available in color online. and plants, which suggests that it represents an ancient process. It is also generally accepted that PTGS, and the related homology-dependent transcriptional gene silencing (TGS), is a defense mechanism of eukaryotes against intrusive genes (transposons, viruses) (Vance and Vaucheret 2001; Waterhouse et al. 2001a). In the past few years, some key elements of the mechanism have been identified.

A central role in PTGS is played by double-stranded (ds)RNA molecules. Expression or introduction of dsRNA in eukaryotic cells can trigger gene silencing of transgenes, endogenes, and viruses in a sequence-specific manner (Montgomery et al. 1998; Sijen and Kooter 2000; Vaucheret and Fagard 2001). Normally, no dsRNA molecules are detectable in an eukaryotic cell and it has become clear that they are both target and trigger for the degradation of homologous RNAs (Vaucheret and Fagard 2001; Voinnet et al. 1999). In mammalian cells, long dsRNA molecules initiate a sequence-unspecific defense mechanism by activating the interferon response, which eventually switches off protein translation (Alexopoulou et al. 2001; Clemens and Elia 1997; Kumar and Carmichael 1998). However, dsRNA is also a replicative intermediate during replication of RNA viruses produced by the viral replicase. Therefore, the presence of dsRNA in plant cells is considered a characteristic of virus infection and has earlier been used as a diagnostic tool (Morris and Dodds 1979). Viral dsRNA is the trigger for induction of the PTGS antiviral defense mechanism in plants (Dalmay et al. 2000). As a response to this, viruses have evolved or adapted genes, which suppress PTGS (Vance and Vaucheret 2001). In cells where PTGS is active, small sequence-specific sense and antisense RNAs of 21 to $23 \mathrm{nt}$ become detectable (Hamilton and Baulcombe 1999; Waterhouse et al. 2001b). Recently, the term "small interfering RNAs" (siRNAs) was coined for these RNAs (Elbashir et al. 2001). They are not only indicative of PTGS, but play a key role in the PTGS mechanism (Waterhouse et al. 2001a).

Cucumber mosaic virus (CMV) is a (+) polarity, singlestranded RNA virus, with segmented genome, and all three genomic RNAs are necessary for systemic infection in plants (Palukaitis et al. 1992). Few sources of natural resistance to this virus are known (Fraser 1986). CMV encodes two virusencoded replicase components ( $1 a$ and $2 a$ ), a capsid protein, a gene responsible for cell to cell movement $(3 a)$, and a suppressor of gene silencing $(2 b)$, which is, however, not active in all CMV-host combinations ( $\mathrm{Ji}$ and Ding 2001). It is anticipated that this suppressor molecule is interfering at a step before the production of the siRNAs (Vance and Vaucheret 2001).

Pathogen-derived resistance strategies can be roughly divided into two groups: those that involve the production of transgenic 
protein (e.g., coat protein, replicase, movement protein-mediated resistance) and those that function at the RNA level only (e.g., sense, antisense, ribozyme, dsRNA-mediated resistance). Pathogen-derived resistance for CMV often showed only partial resistance or very narrow spectrum of resistance to the virus (Beachy 1997). However, high levels of resistance to CMV previously have been reported via replicase-mediated resistance (Anderson et al. 1992), combined expression of the coat protein, and satellite RNA-mediated resistance (Yie et al. 1992). Various levels of resistance to CMV were reported following the transgenic suppression of viral RNA1 (Canto and Palukaitis 1998, 2001), whereas only moderate "resistance" was reported following the introduction of a trans-cleaving ribozyme targeting the conserved leader sequences of CMV RNA 1 and 2 (Kwon et al. 1997).

Here, we report the engineering of transgenic oriental tobacco lines that express dsRNA homologous to the $3^{\prime}$ portion of the CMV RNA3 genome. This manipulation made several trans- genic lines completely resistant to CMV. We demonstrate that the presence of homologous CMV siRNAs in the plants prior to infection can be used as a molecular marker to predict resistance. Plant lines that failed to produce siRNAs at a detectable level were susceptible to CMV infection, whereas plants producing siRNAs were resistant to CMV. Further, we demonstrate that siRNA production is induced in young plants at higher temperatures. Despite the presence of the viral $2 b$ suppressor gene, siRNAs accumulate in the transgenic plants after virus infection.

\section{RESULTS}

Generation

of transgenic tobacco expressing a CMV dsRNA.

In order to express a dsRNA in vivo, we constructed a plasmid with an inverted repeat of a cDNA fragment of the CMV coat protein $(\mathrm{CP})$ gene. We chose as a cDNA unit a 747-bp fragment of the CP gene of a Greek strain of CMV lacking the
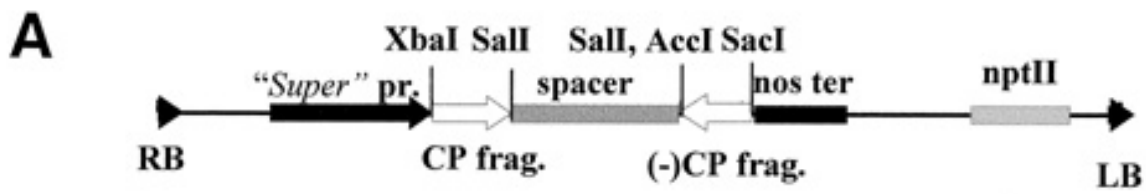

p940CMV

B

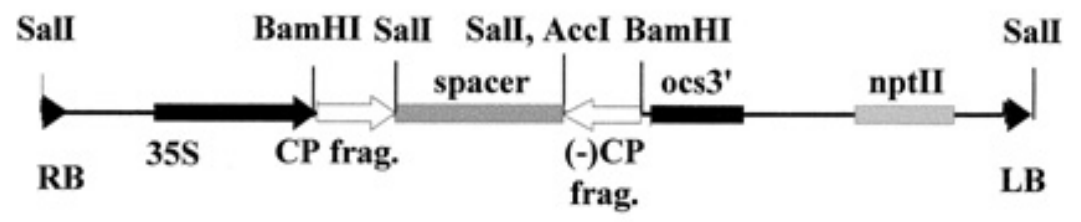

\section{PARTCMV}

Fig. 1. Map of T-DNA fragment of constructs carrying the Cucumber mosaic virus (CMV) coat protein (CP) inverted repeat A, under a "Super" promoter and $\mathbf{B}$, under a $35 \mathrm{~S}$ promoter.
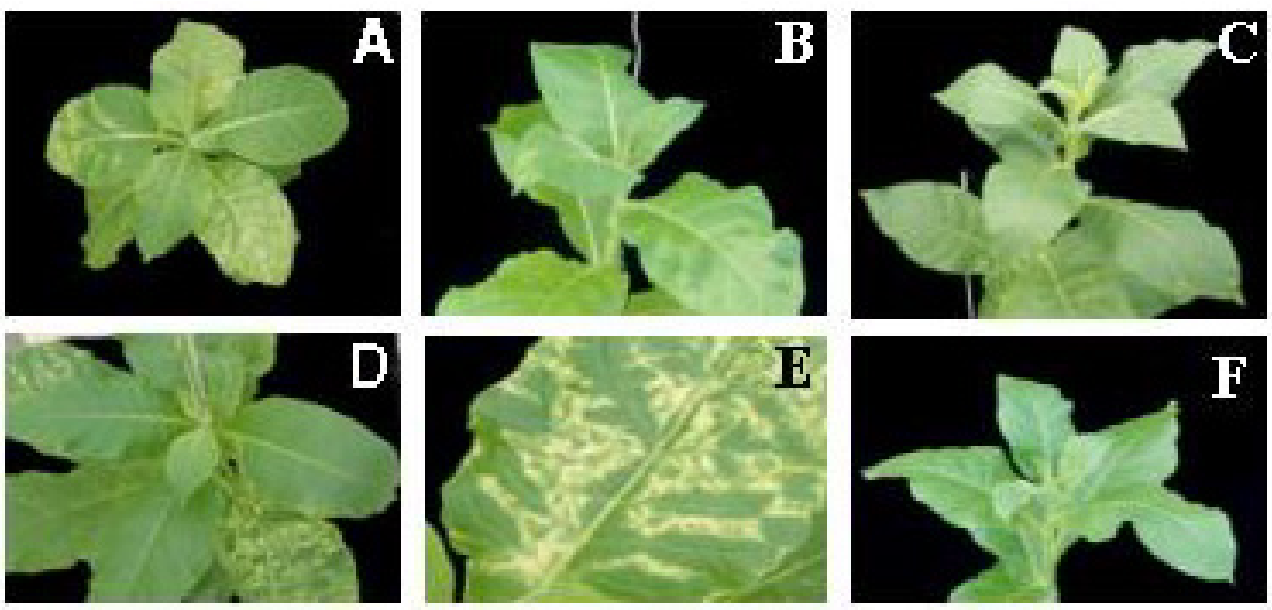

Fig. 2. Phenotype of Cucumber mosaic virus (CMV) infection on CMV-hairpin transgenic tobacco lines. A, Wild-type plant, full symptoms. B, Transgenic plant cmv35/1; very mild symptoms, green mosaic only. Later, this plant converted into a recovery phenotype. C, Transgenic plant $\mathrm{cmv} 40$, recovery phenotype. D, Transgenic plant cmv14, gradual recovery phenotype. E, Transgenic plant cmv14, 'resistant' areas along the veins. F, Transgenic plant cmv13, resistant phenotype. 
initiator AUG codon. Inverted repeats often are instable; therefore, we separated the two cDNA units by an unrelated "spacer" sequence. The spacer has to be sufficiently long to avoid recombination in Escherichia coli. For that purpose, we chose a 1,445-bp fragment of bacteriophage $\lambda$ DNA (a 639-bp spacer was unstable and was not used further). The CMV cDNA unit was fused in sense and antisense orientation to the spacer and introduced in two different plant transformation vectors, pART27 (Gleave 1992), and pATC940. In these two transformation vectors, the hairpin CMV DNA construct was under the control of either a single 35S (Gleave 1992) or the (Aocs) $)_{3}$ Pmas promoter (Ni et al. 1995), referred to here as the "Super" promoter (Fig. 1).

A total of 118 independent transgenic tobacco lines were created via Agrobacterium-mediated transformation. In all, 91 plants belonged to the Super promoter series, where the $\mathrm{CP}-$ CMV hairpin construct was expressed from the Super promoter, and 27 belonged to the $35 \mathrm{~S}$ series. $\mathrm{T}_{1}$ seeds from 54 lines of the Super series and 14 lines of the $35 \mathrm{~S}$ series were germinated on kanamycin selection media, under conditions where wild-type seedlings did not survive. The transgene loci number was estimated and the analysis revealed 47 plants with a single transgenic locus and 21 plants with two or more loci. Thirteen randomly selected $\mathrm{T}_{0}$ transgenic plants also were analyzed for transgene copy number by Southern hybridization, which showed that the transgenic loci number matched with the copy number estimated from germination on kanamycin (data not shown).

\section{Challenging $\mathrm{T}_{0}$ plants with $\mathrm{CMV}$.}

No phenotypic abnormalities were observed in the transformed plants carrying the CP-hairpin construct so that the $\mathrm{T}_{0}$ plants could be challenged with CMV in order to test for possible resistance. For that purpose, $\mathrm{T}_{0}$ plants were inoculated approximately 2 weeks after they were taken out to the greenhouse (six-leaf stage) with fresh inoculum of CMV (strain G). All 118 transgenic lines were challenged with the same inoculum. The response to this challenge was diverse. Susceptibility to CMV infection was scored on the basis of symptom development. Northern analysis confirmed that the concentration of CMV RNA correlated with severity of symptoms (data not shown).

At 10 to 15 days postinoculation (dpi), all wild-type (wt) tobacco showed the characteristic severe CMV mosaic (Fig. 2A). Infected wild-type plants were stunted to approximately two-thirds of the height of control (noninfected) plants. Although infected plants were scored approximately every 14 days, the final classification refers to the state of the plants at the last scoring date, roughly 3 months postinoculation.

Three major categories of plants could be discriminated, which will be described further.

As detailed in Table $1,85 \mathrm{~T}_{0}$ transgenic lines were susceptible to CMV. However, only approximately one-third of those plants reacted with symptoms as intense as the wild-type plants. The residual plants showed greatly variable degrees of symptom expression, including those that reacted with very mild symptoms (e.g., very mild green mosaic in fully developed leaves) (Fig. 2B). Plants that reacted mildly were much larger in size than CMV-infected control plants. Also, the tem- poral pattern of symptoms following infection varied among individual transgenic plants. The majority of the susceptible plants showed symptoms during 10 to 15 days dpi, but there were plants that did not show the first CMV symptoms until 55 dpi. Four plants classified as susceptible showed partial recovery (i.e., no symptoms) in three or more leaves and, in one case, stayed free of symptoms for 50 days, before symptoms reappeared in the newly emerging leaves. These transgenic plants appeared as "three zone" plants, having symptoms in the lower and upper zone and being symptom-free in the middle zone. From this description, it is evident that a significant portion of the plants showed a variable degree of protection against CMV despite being classified as susceptible.

The second group of transgenic plants responded to challenge by CMV with a "recovery" phenotype (Fig. 2C and D). These are plants that responded initially with CMV symptoms of variable severity. However, at a later stage, these plants formed young leaves that were completely free of symptoms. Only plants that did not show any symptoms in the upper four leaves were grouped into this category. It should also be added that wt plants sometimes respond with a decrease in symptoms in the upper leaves. Occasionally, they can even form a leaf that is symptom free, but we never observed a wt plant where more than one, let alone four, leaves became symptom free.

In some transgenic plants where CMV symptoms appeared, including plants that eventually showed recovery, different types of symptoms were observed in different leaves of the same individual. Lower leaves showed the severe wt CMV symptoms; whereas, in upper leaves, symptoms gradually became milder (Fig. 2D). In some cases, symptoms were more pronounced between primary and secondary veins, leaving most of the vein and adjacent tissue symptom free (Fig. 2E). When plants that showed such mild symptoms in their upper leaves were grown further, the newly developing leaves were in some cases symptom free; whereas, in other plants, emerging leaves-developed wt CMV symptoms.

The third group of plants was resistant (i.e., completely devoid of any disease symptoms throughout the entire testing period). In order to confirm that these plants were virus free, we used extracts of those plants to inoculate young wt tobacco plants. This sensitive bioassay confirmed that the plants were devoid of any infectious CMV (data not shown).

\section{Correlation of copy number of the transgenes and resistance phenotype.}

In view of the variable response of the $T_{0}$ plants to $C M V$, we analyzed whether there is a correlation between resistance and copy number of the hairpin transgene. $\mathrm{T}_{0}$ plants of 37 lines

Table 2. Relation between transgene loci number and resistance to Cucumber mosaic virus (CMV) in $\mathrm{T}_{0}{ }^{\mathrm{a}}$

\begin{tabular}{lcc}
\hline $\begin{array}{l}\text { Response to } \\
\text { CMV inoculation }\end{array}$ & $\begin{array}{c}\text { Single transgene } \\
\text { locus }\end{array}$ & $\begin{array}{c}\text { Two or more } \\
\text { transgenic loci }\end{array}$ \\
\hline Resistant & 6 & 4 \\
Susceptible & 22 & 5 \\
\hline
\end{tabular}

${ }^{a}$ Data from $37 \mathrm{~T}_{0}$ lines.

Table 1. Response of Cucumber mosaic virus (CMV)-hairpin transgenic $\mathrm{T}_{0}$ plants to CMV virus inoculation

\begin{tabular}{|c|c|c|c|c|c|c|c|c|}
\hline \multirow[b]{2}{*}{ Response } & \multicolumn{2}{|c|}{ Total transgenic plants } & \multicolumn{2}{|c|}{ "Super" promoter } & \multicolumn{2}{|c|}{$35 \mathrm{~S}$ promoter } & \multicolumn{2}{|c|}{ Wild-type controls } \\
\hline & Number & $\%$ & Number & $\%$ & Number & $\%$ & Number & $\%$ \\
\hline Resistant & 20 & 17 & 15 & 16 & 5 & 18 & 0 & 0 \\
\hline Recovered & 13 & 11 & 9 & 10 & 4 & 15 & 0 & 0 \\
\hline Susceptible & 85 & 72 & 67 & 74 & 18 & 67 & 8 & 100 \\
\hline Total & 118 & 100 & 91 & 100 & 27 & 100 & 8 & 100 \\
\hline
\end{tabular}


were analyzed for their segregation pattern. As can be seen from the data in Table 2, $\mathrm{T}_{0}$ lines with two or more transgene loci were more likely to become resistant.

\section{Analysis of CMV-specific RNAs in $\mathrm{T}_{0}$ transgenic plants.}

Representative plants of the resistant and susceptible phenotypic groups were examined for CMV-specific transcripts originating from the transgene on a Northern blot (Fig. 3). The transgene contains the $\mathrm{CP}$ gene in both sense and antisense orientation; therefore, we used a sense RNA probe to avoid crosshybridization with the great majority of viral sequences. As an internal standard, we used a probe specific for actin mRNA (Fig. 3, lower row). We found that the content of the hairpin transcript was variable in individual transgenic lines (Fig. 3, upper row), including groups of plants that responded in the same way to the virus; for example, resistant plants. In the resistant line cmv35/2, the CMV-specific transcript could be detected, whereas resistant line cmv28 contained only traces of the hairpin RNA. Northern blots represent the steady state of the RNA concentration, but not the transcription rate; this could indicate that, in cmv28, the dsRNA became quickly degraded due to the activation of PTGS.

The presence of sense and antisense RNA molecules of 21 to 23 nts-now termed siRNA-is characteristic of PTGS (Hamilton and Baulcombe 1999); therefore, noninfected clones of two susceptible and two resistant transgenic $\mathrm{T}_{0}$ lines were analyzed for the presence of such CMV homologous RNAs. The presence of siRNAs would indicate whether the PTGS response had been activated, especially in plants like cmv28 where transcript levels of the full-length hairpin had been low. The analysis showed that only in the resistant plants cmv28 and cmv35/2 could the characteristic short RNAs be detected (Fig. 4). It is interesting to note that, in plant cmv28, the concentration of siRNAs was slightly higher when normalized to the U1 RNA probe (Fig. 4). In accordance with this, the concentration of the full hairpin transcript was lower (Fig. 3).

We also examined the occurrence of siRNA in transgenic plants challenged with the virus. Approximately 1 month after inoculation with $\mathrm{CMV}$, six different $\mathrm{T}_{0}$ transgenic plants were analyzed using sense CMV CP probes. The ds nature of the short RNAs was confirmed by hybridizing also with the antisense CMV CP probe (data not shown). All transgenic and wt plants infected with CMV and showing symptoms, contained CMV-homologous siRNA (Fig. 5). However, after challenge with CMV, the level of siRNAs correlated with neither the intensity of the symptoms (for example, 1, 2, and 4 in Fig. 5) nor the levels of total viral RNA present (data not shown) in the infected plant.

\section{The generation of CMV-specific siRNAs}

from the hairpin transgene construct correlates with resistance to $\mathrm{CMV}$ infection.

In view of the observation that the two $\mathrm{T}_{0}$-resistant plants analyzed in Figure 4 showed short CMV-specific RNAs prior to the inoculation with CMV, we wanted to test whether that was a general correlation. Offspring of 20 transgenic lines (cmv2, cmv3, cmv4, cmv5, cmv6, cmv9, cmv13, cmv16, cmv19, cmv20, cmv22, cmv24, cmv25, cmv26, cmv28 cmv34, cmv36, cmv37, cmv42, and cmv65) were analyzed for the presence of $\mathrm{CMV}$-specific siRNAs prior to inoculation with CMV, which occurred at approximately the seven-leaf stage. For each transgenic $\mathrm{T}_{0}$ plant, two $\mathrm{T}_{1}$ offspring were selected randomly after kanamycin selection. Between $T_{1}$ siblings, no difference was observed regarding presence or amount of CMV-specific siRNAs, indicating that each transgenic line has a distinct expression pattern for short CMV-specific RNAs. CMV-specific siRNAs could not be detected for cmv2, cmv6, cmv9, cmv22, cmv24, cmv26, cmv34, cmv36, and cmv42. Nine of these had a single copy of the CP-hairpin transgenic locus and only one (cmv34) had multiple transgene loci. In contrast, CMV-specific siRNAs were detected for cmv3, cmv4, cmv5, cmv13, cmv16, cmv19, cmv20, cmv25, cmv28, cmv37, and cmv65, in different amounts (Fig. 6). Five of these (cmv3, cmv5, cmv16, cmv20, and cmv37) had a single copy of the CP-hairpin transgenic locus and six (cmv4, cmv13, cmv19 cmv25, cmv28, and cmv65) had multiple transgene loci.

All plants where CP-CMV-specific siRNA could not be detected were offspring of susceptible parents. In contrast, plants where siRNAs were detected were offspring of $\mathrm{T}_{0}$-resistant plants. The only exceptions were cmv20, which showed in $\mathrm{T}_{0}$ a recovery phenotype but was found susceptible in $T_{1}$, and cmv4, which was susceptible in $\mathrm{T}_{0}$ but showed recovery in $\mathrm{T}_{1}$. When the $T_{1}$ plants were challenged with CMV, the relation between the presence of siRNAs and resistance was even stronger than in the $T_{0}$ generation. $T_{1}$ plants that produced siRNAs proved to be resistant to the virus. In contrast, in susceptible plants, CMV-specific siRNAs has not been detected. An exception to this rule was again plant line cmv20, which produced siRNA at low level but was susceptible to CMV infection in $\mathrm{T}_{1}$. The correlation between the presence of CMV-

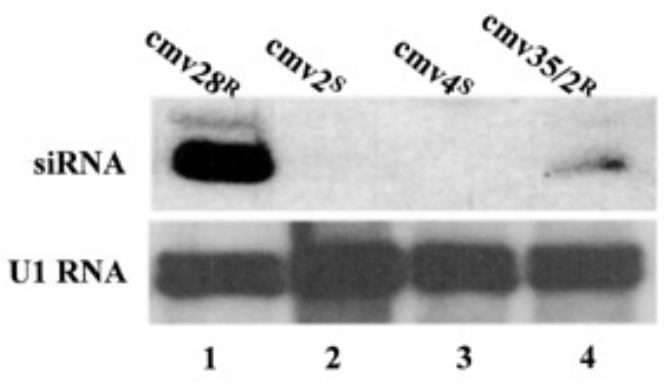

Fig. 4. Detection of Cucumber mosaic virus (CMV)-specific small interfering RNAs in noninfected $\mathrm{T}_{0}$ transgenic plants. Northern hybridizations with a sense $\mathrm{CMV}$-coat protein (CP) probe (top) and a probe specific to U1 RNA (bottom).

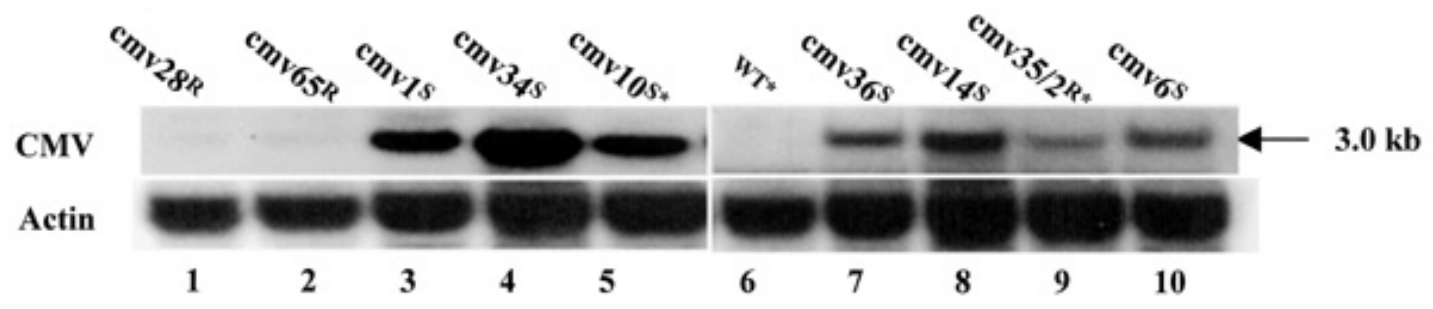

Fig. 3. Hairpin transcription levels detected in $\mathrm{T}_{0}$ transgenic plants. Northern hybridization with a Cucumber mosaic virus coat protein (CMV-CP) sense probe, specific for CMV antisense RNA (top) and a probe specific for actin mRNA (bottom). WT = wild-type plant; R = resistant plant; $\mathrm{S}=$ susceptible plant; * = plants that had been challenged with CMV before RNA extractions 
specific siRNAs present in $T_{1}$ and resistance to the virus is summarized in the lower panel of Figure 6. Occurrence of CMV-specific siRNA rather than steady-state concentration of the full-length CMV hairpin RNA transcript is indicative for the resistant or susceptible phenotype. From these data, it is also evident that the level of siRNAs is not necessarily influenced by the copy number; for example, line cmv37 produced high levels of siRNA from a single transgene.

\section{Transgene-specific siRNA concentration can be influenced by temperature and developmental stage.}

We were interested to see whether environmental and developmental factors could influence the amount of the siRNAs present. For this purpose, two $\mathrm{T}_{1}$ clones each of lines cmv16 and cmv28 were used to test the influence of temperature on the concentration of siRNAs. These lines were chosen because they seemed to represent the two extremes of resistant lines: cmv28 had multiple-transgene loci and very high levels of siRNA, whereas cmv16 had single transgene loci and showed only very low levels of siRNA when analyzed at the seven-leaf stage. One clone of each line was grown in a growth chamber at $25^{\circ} \mathrm{C}$ and the other at $32^{\circ} \mathrm{C}$. RNA samples were extracted at two time points, at 20 and 30 days postgermination (dpg) (approximately five- and seven-leaf stage). At the early time point, siRNAs could be detected in cmv 28 extracts of plants grown at both 25 and $32^{\circ} \mathrm{C}$ although, at $32^{\circ} \mathrm{C}$, CMV siRNAs were found with a

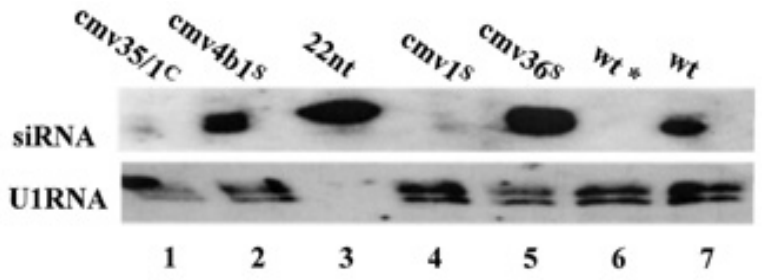

Fig. 5. Detection of coat protein Cucumber mosaic virus (CP-CMV)-specific small interfering RNAs from infected $\mathrm{T}_{0}$ plants 14 days post-CMV infection. Northern hybridizations of representative plants with a CMV CP sense probe (top) and a probe specific to U1 RNA (bottom), which was taken as a reference because it is a relatively small (156 bases), and less structured RNA present in all eukaryotic cells. Samples were taken from plants with different CMV symptoms: severe (cmv36), intermediate (cmv1), mild (cmv4b1), and mild symptoms that eventually recovered $(\mathrm{cmv} 35 / 1) . \mathrm{Wt}=$ wild-type plant, $*=$ plant not challenged with the virus used as a control, $\mathrm{C}=$ recovered plant, $\mathrm{S}=$ susceptible plant, $22 \mathrm{nts}=22$ nucleotide-size marker. significantly higher concentration at a higher temperature. In line cmv16, siRNA could be detected only in the plant grown at $32^{\circ} \mathrm{C}$ but not at $25^{\circ} \mathrm{C}$. The analysis of samples taken at the second time point $(30 \mathrm{dpg})$ revealed CMV siRNAs in both lines. However, at higher temperature, siRNAs were detected in both plant lines at a higher concentration (Fig. 7).

From the above, it is also evident that the siRNA concentration rose between the five-leaf and seven-leaf stages, although the speed of accumulation varied between the two plants tested here. The cmv28 uninfected transgenic plant, showing high level of siRNAs, was further analyzed at later developmental stages in the greenhouse. RNA was extracted from four leaves of cmv28. Leaves from this plant were taken in such a way as to ensure the widest possible leaf age range of a mature tobacco plant ( 2 months old). No significant differences in the siRNA concentration were observed between leaves of different age for this plant; thus, it seems that, from the seven-leaf stage on, the concentration reached a plateau that remained stable in the course of further development (Fig. 8).

\section{DISCUSSION}

We report here the engineering of transgenic tobacco lines that express an RNA derived from the CMV CP that is able to form an intramolecular ds structure. The introduction of this CMV-specific dsRNA transgene made a significant portion of the transgenic lines completely resistant to CMV, demonstrating that dsRNA-mediated protection is an effective strategy to engineer cucumovirus-resistant plants. It should be stressed that transgenic plants grouped as resistant in this study are plants fully resistant to $\mathrm{CMV}$, as estimated from phenotype, Northern hybridization, and bioassay.

The suppression of viruses upon introduction of homologous ds sequences into plants has been previously reported (Smith et al. 2000; Wang et al. 2000). It is believed that the presence of the artificially expressed dsRNA activates the plant's PTGS machinery even in the absence of the virus. In that sense, the artificially introduced dsRNA mimics the presence of a virus. We could show that siRNAs that are indicative of activated PTGS are produced in a significant number of the transgenic lines, directly demonstrating that the artificially expressed dsRNA was indeed, in those lines, subject to RNA silencing. According to the model of PTGS, siRNAs are incorporated into the RNA-induced silencing complex (RISC), which is responsible for the actual RNA degradation of the homologous target RNA (Hammond et al. 2000).

\section{'cmv' lines}

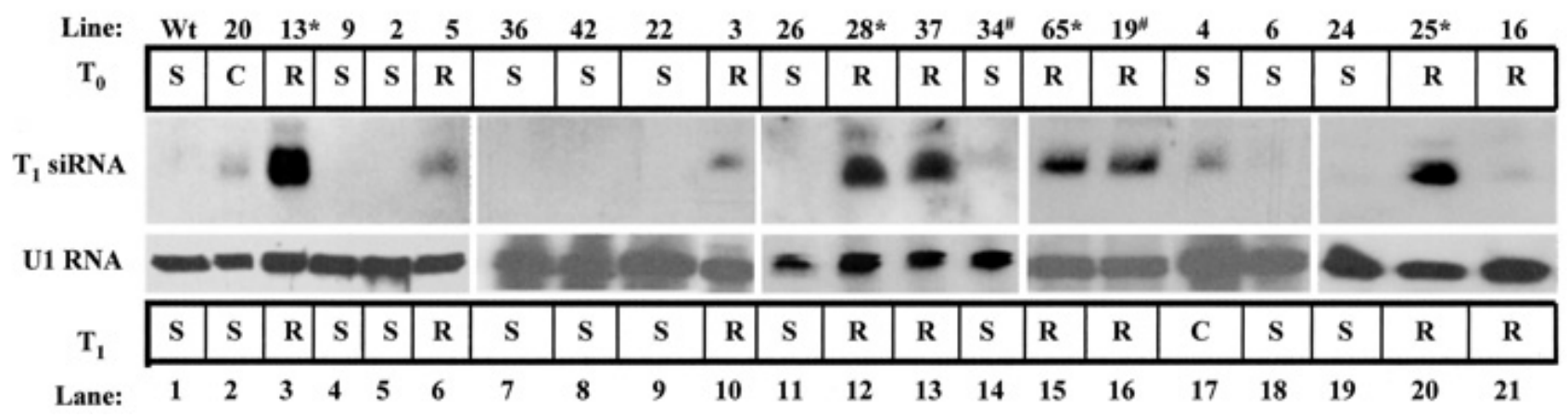

Fig. 6. Presence of Cucumber mosaic virus coat protein (CMV-CP)-specific small interfering (si)RNAs before infection is indicative of resistance to the CMV virus. Top: names of the cmv lines and their response to challenge with CMV at $\mathrm{T}_{0}$. Bottom: detection of siRNAs from $\mathrm{T}_{1}$ plant extracts before infection with CMV and hybridization for U1 RNA. The lower row indicates the phenotype after challenge of $\mathrm{T}_{1}$ plants with $\mathrm{CMV}$. $\mathrm{S}=$ susceptible to the virus, $\mathrm{R}=$ resistant to the virus, $\mathrm{C}=$ recovered following $\mathrm{CMV}$ infection, $*=$ lines that carry multiple transgene loci in both $\mathrm{T}_{0}$ and $\mathrm{T}_{1}$, \# = lines that carried multiple transgene loci in $\mathrm{T}_{0} ; \mathrm{T}_{1}$ copy number data not available. 
In a CMV-infected wt tobacco, virus and plant seem to be in a fragile balance: the plant suppresses the virus via PTGS (and possibly other mechanisms) and the virus reacts by rapid replication and suppression of the host's silencing response (Kasschau and Carrington 1998; Voinnet et al. 1999). The availability of a RISC complex loaded with CMV siRNAs prior to the invasion of the virus shifts the battle to the favor of the host. The plant host has the possibility of an instant and massive attack of a homologous newly invading viral RNA, as the PTGS response machinery is already activated. The attack could be the direct degradation of viral RNA or prevention of CMV expressing its PTGS suppressor protein fast enough and in sufficient quantities. It is known that cucumoviruses, including CMV, contain at least one such suppressor, the gene $2 b$ (Beclin et al. 1998). The $2 \mathrm{~b}$ protein is a protein that contains a nuclear localization signal required for long distance viral movement in some hosts and it suppresses silencing only in newly emerged tissues that develop after infection (Brigneti et al. 1998). Our data show that this viral counter defense is not sufficient when the virus invades a host plant with already activated PTGS.

We could provide evidence that the presence and concentration of siRNAs immediately prior to challenge by the virus plays a decisive role for the resistance of the plant to the virus. Plants that produced high levels of siRNAs were resistant to inoculations with highly infectious extracts. Thus, the occurrence of short RNAs can be taken as a molecular marker to predict the degree of resistance, a clear advantage of dsRNAmediated protection strategy. However, plants devoid of any preformed siRNAs also showed reduced susceptibility to viral infection, as evidenced by the ameliorated disease symptoms. The most reasonable explanation is that, in those plants, the complete PTGS process is not yet fully activated, at least not to levels sufficient to fully suppress an invading virus. On the other hand, even in those plants that do not contain detectable levels of short RNAs, the premade CMV dsRNA supports the PTGS response even after a virus is established in the host. Most likely, the PTGS response is more intense or more rapid than in a wild-type plant. In a general sense, the strategy to express a virus-derived dsRNA has is a striking similarity to an immunization. Here, we are delivering to the host organism pathogen-derived "antigens", which are, in our case, pathogenderived dsRNA. The exposure to this "antigen" causes protection for the host. The analogy can be extended as the dsRNA antigen is fragmented into smaller antigen units.

It remains a challenge to find out why only some of the transgenic lines generate substantial amounts of siRNA while others do not. The variability is reminiscent of the variability when antisense genes had been introduced for gene suppression (Tabler 1993). In retrospect, this similarity may not be surprising, because antisense RNA inhibition is done by activation of PTGS (Di Serio et al. 2001). In neither case can a fully satisfactory explanation be given. For antisense genes, a "position effect" depending on the integration site into the genome already has been discussed. Here, we could show that the strength of the promoter seems to be less important. Moreover, the different response (production of siRNA and resistance) does not seem to depend on the accumulation of the hairpin dsRNA alone. We could identify plants with high levels of hairpin transcript, but devoid of siRNAs (e.g., cmv36, Figs. 3 and 5; or cmv6, Figs. 3 and 6,). However, we have not monitored how far transcription rates are influenced by the sequence context or whether some of the transgene coding sequences have been methylated. In addition, a number of the susceptible lines which have not been analyzed for their steady state transcriptional level might be methylated at their promoter regions, so that they undergo transcriptional silencing. We could show, however, that the likeliness of generating CMV-specific short RNAs increases with the copy number of the transgene. This is not surprising. So far, we do not know whether more than one transgene has a cooperative effect or whether the production of short RNAs is coupled to a particular locus. Segregation studies will clarify this question. This does not exclude, as we have shown, the possibility that there are additional environmental factors. For example, we found that elevated growth temperature favor the formation of siRNAs. This is in agreement with general reports on the influence of developmental and environmental factors on PTGS in plants (Kunz et al. 1996; Pang et al. 1996; Vaucheret et al. 1995). Meza and associates (2001) recently reported a strong impact of environmental factors on transcriptional silencing of Arabidopsis siblings. Temperature-dependent PTGS has been reported also for RNA interference (RNAi) in Drosophila (Fortier and Belote 2000). Such small environmental influences are most likely responsible for shifting recovery and resistant phenotypes as we observed. Also, the developmental stage of the leaf at the time of inoculation may play a role in shifting the fragile balance.

Regardless of the mechanistic details, the expression of viral dsRNA seems to be a highly efficient way to engineer virus-resistant plants. The frequency of the occurrence of resistant lines is high. Most importantly, the monitoring of short RNAs makes it possible to select for the most promising lines that are highly resistant to the virus. This strategy offers significant advantages compared with previous efforts in engineering virusresistant pants. cmv28

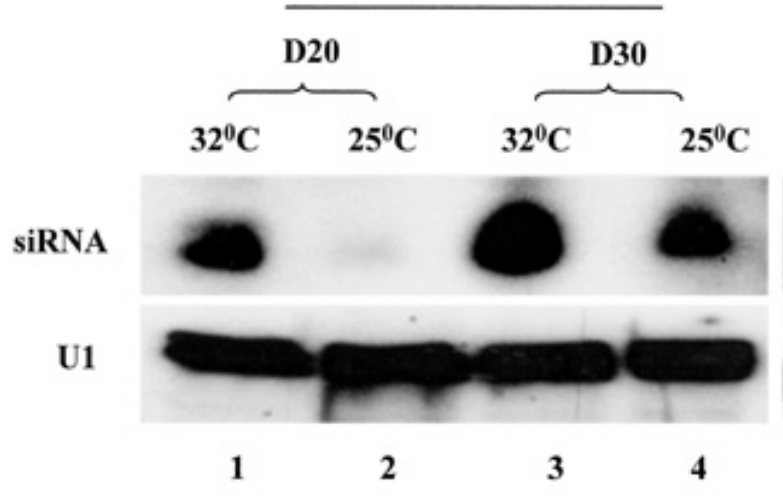

emv16

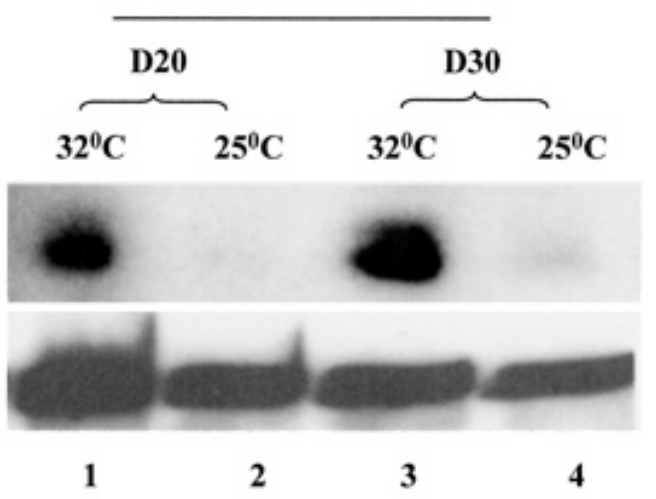

Fig. 7. Temperature effect on small interfering RNAs of coat protein Cucumber mosaic virus (CP-CMV) hairpin transgenic plants cmv28 and cmv16. Northern hybridizations with a CMV CP sense probe. $\mathrm{D}=$ days postgermination. 


\section{MATERIALS AND METHODS}

\section{Cloning and construction}

of plasmid producing hairpin RNA.

An 883-bp fragment from RNA 3 of CMV virus (strain GR 21 ) containing the $\mathrm{CP}$ gene was cloned by reverse transcription polymerase chain reaction in a pBluescript $\mathrm{KS}$ vector (Stratagene). The presence of the $\mathrm{CP}$ sequence was confirmed by sequencing. In order to avoid production of $\mathrm{CP}$ protein in the transgenic plants, we deleted a 136-bp SalI fragment in the $5^{\prime}$ end (CP-43). The resulting 747-bp fragment contained the 718-bp 3' end of the $C P$ gene. A 1,445-bp AccI $\lambda$ phage DNA fragment (nucleotides 31,301 to 32,745 on the $\lambda$ DNA) was used to separate the $C P$ gene sequences in the inverted repeat as shown on Figure 1. This construct was introduced in a plasmid pART27 (Gleave 1992) under the control of a 35S promoter and in plasmid pATC940 under a (Aocs) ${ }_{3}$ Pmas promoter. (Ni et al. 1995)

\section{Plant material and transformation.}

Leaf discs for transformation experiments were excised from young leaves of Nicotiana tabacum 'Basmas' (N34/4 of the National Tobacco Institute of Greece) grown in the greenhouse. Explants and plants in the growth chamber were grown at $25^{\circ} \mathrm{C}$ day and $18^{\circ} \mathrm{C}$ night with a 16-hr photoperiod provided by cool white fluorescent tube lights to give $90 \mu \mathrm{mol} \mathrm{m} \mathrm{m}^{-2}$ photosynthetically active radiation. Following co-cultivation with Agrobacteria (Agrobacterium tumefaciens, strain LBA4404), discs were placed on Murashige-Skoog (MS) media containing hormones (BA, $1 \mathrm{mg} / \mathrm{liter}$, and NAA, $0.1 \mathrm{mg} / \mathrm{liter}$ ) and antibiotics (kanamycin, $100 \mathrm{mg} / \mathrm{liter}$, and cefotaxime, $250 \mathrm{mg} / \mathrm{liter}$ ) in a growth chamber. Shoots regenerating from the explants then were transferred on MS media (Murashige and Skoog 1962) with antibiotics (kanamycin, $100 \mathrm{mg} / \mathrm{liter}$, and cefotaxime, 250 $\mathrm{mg} / \mathrm{liter}$, but without hormones in order to initiate rooting. Plantlets were then transferred to nonaseptic conditions and were grown to maturity in the greenhouse with cabinets that were temperature controlled using a commercial air-conditioning system at $22^{\circ} \mathrm{C}$ day and $18^{\circ} \mathrm{C}$ night.

A total of 118 lines were generated, 91 lines with the CPCMV hairpin under the Super promoter, which include cmv1, cmv2, cmv3, cmv4, cmv4b1, cmv6, cmv9, cmv10, cmv14, cmv19, cmv20, cmv22, cmv24, cmv25, cmv26, cmv28, cmv34, cmv36, cmv42, and cmv65; and 27 lines under the $35 \mathrm{~S}$ promoter, which include cmv35/1 cmv35/2.

\section{$T_{1}$ seed germination and scoring.}

Approximately $40 \mathrm{~T}_{1}$ seeds of each $\mathrm{T}_{0}$ transgenic plant were surface sterilized $\left(10 \% \mathrm{Na}_{3} \mathrm{PO}_{4}\right.$ for $15 \mathrm{~min}, 70 \% \mathrm{ETOH}$ for 1 min, and $10 \%$ commercial bleach for $20 \mathrm{~min}$ ), washed three times with sterile distilled water, and then sown on plates containing MS selection media (kanamycin at $125 \mathrm{mg} / \mathrm{liter}$ ).
Transgenic locus copy number was calculated on the basis of the ratio of growing to necrotic seedlings approximately 15 days post germination.

\section{Plant infections.}

CMV-infected fresh tobacco leaf $(0.1 \mathrm{~g})$ was ground in 0.1 $\mathrm{ml}$ of phosphate buffer $\left(5 \mathrm{mM} \mathrm{KH} \mathrm{PO}_{4}, 6.25 \mathrm{mM} \mathrm{Na}_{2} \mathrm{HPO}_{4}\right.$, $\mathrm{pH}$ 6.8) and was used to infect a leaf of young tobacco plantlets approximately at the six-leaf stage. Typical CMV mosaic symptoms appeared on the susceptible plants 8 to $12 \mathrm{dpi}$.

\section{Hybridization analyses.}

RNA from plants 6 or more weeks old was isolated always from a single fresh leave of fresh weight 0.6 to $0.8 \mathrm{~g}$. RNA from younger plants was isolated from more than one fresh leaf of a total weight of at least $0.6 \mathrm{~g}$. Total RNA was isolated with acid phenol ( $\mathrm{pH}$ 4.0) and small RNA enrichment was achieved by resuspension of the RNA pellet in $8 \mathrm{M} \mathrm{LiCl}$ (Papaefthimiou et al. 2001). Total RNA $(10 \mu \mathrm{g})$ was separated by electrophoresis on denaturing agarose gels; whereas, for the detection of siRNAs, $25 \mu \mathrm{g}$ of total RNA was used for the $\mathrm{LiCl}$ fractionation and the whole sample was heat treated in formamide buffer and loaded onto a $12 \%$ polyacrylamide slab gel (12\% acrylamide, $0.6 \%$ bisacrylamide) containing $7 \mathrm{M}$ urea and $50 \mathrm{mM}$ TBE buffer (50 mM Tris, $41.5 \mathrm{mM}$ boric acid, 0.5 mMEDTA) and separated by electrophoresis.

Nucleic acid transfer from agarose gels onto nylon membranes was performed by capillary transfer according to standard procedures (Sambrook et al. 1989). RNAs were transferred from acrylamide gels to nylon membrane (Nytran N membrane; Schleicher and Schuell, Dassel, Germany) and fixed by UV cross-linking.

Prehybridization and hybridization, which included the RNA probe at approximately $10^{6} \mathrm{cpm}$ per $\mathrm{ml}$, was carried out in $5 \times$ $\mathrm{SSC}(1 \times \mathrm{SSC}$ is $0.15 \mathrm{M} \mathrm{NaCl}$ plus $0.015 \mathrm{M}$ sodium citrate), $1 \times$ Denhardt solution, $1 \%$ sodium dodecyl sulfate (SDS), and tRNA carrier at $0.25 \mathrm{mg} / \mathrm{ml}$. RNA sense or antisense probes were used for all hybridizations. Actin hybridization using a soya antisense actin probe was used as an internal standard for transcript Northern hybridizations. Hybridization to U1 RNA (approximately $156 \mathrm{bp}$, a member of the RNAs present in snRNP complexes) using a potato U1 probe was used as an internal standard for "short RNA" Northern hybridizations.

Transcript Northern hybridizations were performed at $65^{\circ} \mathrm{C}$, $50 \%$ formamide., For "small RNA" Northern hybridizations, the temperature was decreased to $53^{\circ} \mathrm{C}$ and no formamide was added. Posthybridization membranes were washed twice with $2 \times$ SSC and $0.2 \%$ SDS for $20 \mathrm{~min}$ at the hybridization temperature. Transcript RNA Northern blots were additionally washed with $0.1 \times \mathrm{SSC}$ and $0.2 \% \mathrm{SDS}$ at the hybridization temperature for $15 \mathrm{~min}$.
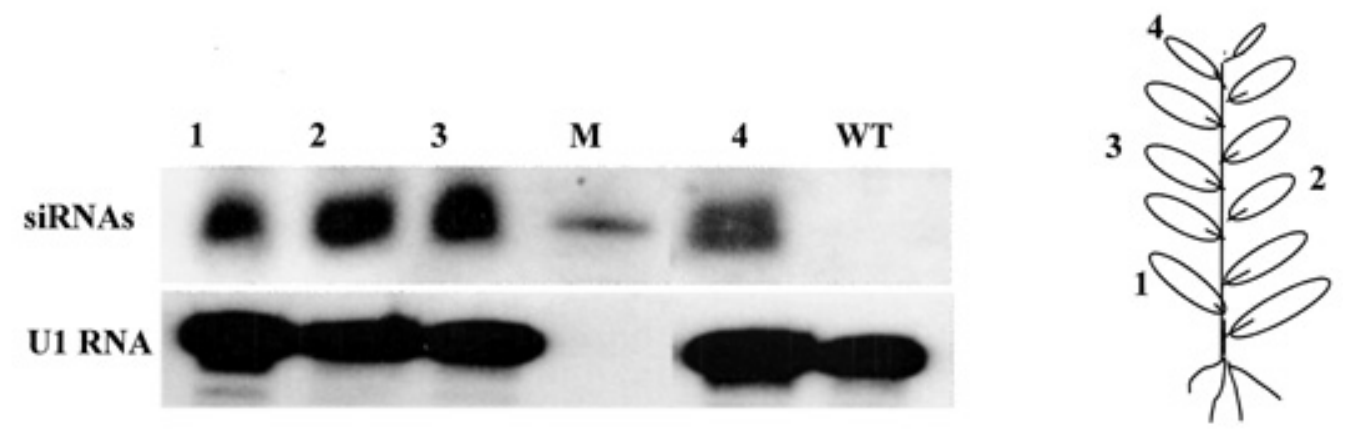

Fig. 8. Cucumber mosaic virus coat protein (CMV-CP)-specific small interfering RNAs of successive leaves in a 2-month-old transgenic tobacco plant cmv28. Lanes 1 to 4: RNA from leaves 1 to 4 ; leaf $1=$ oldest and leaf 4 = youngest. Lane M: 22 nt marker. 


\section{ACKNOWLEDGMENTS}

We thank N. Panopoulos and L. Comai for the vector pATC940, A. Gleave for vector pART27, A. Missiou for technical assistance in Northern hybridizations, P. Kyriakopoulou and T. Sklavounos for the Greek CMV strains, and the Greek Tobacco Institute for kindly donating tobacco seed. This work was supported by grants Tab-Res-Info 96/T/18 and PENED 99ED404 of the European Union and the Greek Ministry of Development, respectively.

\section{LITERATURE CITED}

Alexopoulou, L., Holt, A. C., Medzhitov, R., and Flavell, R. A. 2001. Recognition of double-stranded RNA and activation of NF-kappaB by Tolllike receptor 3. Nature 413:732-738.

Anderson, J. M., Palukaitis, P., and Zaitlin, M. 1992. A defective replicase gene induces resistance to cucumber mosaic virus in transgenic tobacco plants. Proc. Natl. Acad. Sci. U.S.A. 89:8759-8763.

Beachy, R. N. 1997. Mechanisms and applications of pathogen-derived resistance in transgenic plants. Curr. Opin. Biotechnol. 8:215-220.

Beclin, C., Berthome, R., Palauqui, J. C., Tepfer, M., and Vaucheret, H. 1998. Infection of tobacco or Arabidopsis plants by CMV counteracts systemic post-transcriptional silencing of nonviral (trans)genes. Virology 252:313-317.

Brigneti, G., Voinnet, O., Li, W. X., Ji, L. H., Ding, S. W., and Baulcombe, D. C. 1998. Viral pathogenicity determinants are suppressors of transgene silencing in Nicotiana benthamiana. EMBO (Eur. Mol. Biol. Organ.) J. 17:6739-6746.

Canto, T., and Palukaitis, P. 1998. Transgenically expressed cucumber mosaic virus RNA 1 simultaneously complements replication of cucumber mosaic virus RNAs 2 and 3 and confers resistance to systemic infection. Virology 250:325-336.

Canto, T., and Palukaitis, P. 2001. A cucumber mosaic virus (CMV) RNA 1 transgene mediates suppression of the homologous viral RNA 1 constitutively and prevents CMV entry into the phloem. J. Virol. 75:91149120.

Clemens, M. J., and Elia, A. 1997. The double-stranded RNA-dependent protein kinase PKR: Structure and function. J. Interferon Cytokine Res. 17:503-524.

Dalmay, T., Hamilton, A., Rudd, S., Angell, S., and Baulcombe, D. C. 2000. An RNA-dependent RNA polymerase gene in Arabidopsis is required for posttranscriptional gene silencing mediated by a transgene but not by a virus. Cell 101:543-553.

Dellaporta, S. L., Wood, J., and Hicks, J. B. 1983. Isolation of DNA from higher plants. PMB Rep. 4:19-421.

Di Serio, F., Schob, H., Iglesias, A., Tarina, C., Bouldoires, E., and Meins, F., Jr. 2001. Sense- and antisense-mediated gene silencing in tobacco is inhibited by the same viral suppressors and is associated with accumulation of small RNAs. Proc. Natl. Acad. Sci. U.S.A. 98:6506-6510.

Dougherty, W. G., Lindbo, J. A., Smith, H. A., Parks, T. D., Swaney, S., and Proebsting, W. M. 1994. RNA-mediated virus resistance in transgenic plants: Exploitation of a cellular pathway possibly involved in RNA degradation. Mol. Plant-Microbe Interact. 7:544-552.

Elbashir, S. M., Lendeckel, W., and Tuschl, T. 2001. RNA interference is mediated by 21- and 22-nucleotide RNAs. Genes Dev. 15:188-200.

Fortier, E., and Belote, J. M. 2000. Temperature-dependent gene silencing by an expressed inverted repeat in Drosophila. Genesis 26:240-244.

Fraser, R. S. S. 1986. Genes for resistance to plant viruses. Crit. Rev. Plant Sci. 3:257-294.

Gleave, A. P. 1992. A versatile binary vector system with a T-DNA organizational structure conducive to efficient integration of cloned DNA into the plant genome. Plant Mol. Biol. 20:1203-1207.

Hamilton, A. J., and Baulcombe, D. C. 1999. A species of small antisense RNA in posttranscriptional gene silencing in plants. Science 286:950-2.

Hammond, S. M., Bernstein, E., Beach, D., and Hannon, G. J. 2000. An RNA-directed nuclease mediates post-transcriptional gene silencing in Drosophila cells. Nature 404:293-296.

Ji, L.-H., and Ding, S.-W. 2001. The suppressor of transgene RNA silencing encoded by Cucumber mosaic virus interferes with salicylic acidmediated virus resistance. Mol. Plant-Microbe Interact. 14:715-724.

Kasschau, K. D., and Carrington, J. C. 1998. A counterdefensive strategy of plant viruses: Suppression of posttranscriptional gene silencing. Cell 95:461-470.

Kooter, J., Matzke, M., and Meyer, P. 1999. Listening to the silent genes: Transgene silencing, gene regulation and pathogen control. Trends Plant Sci. 4:340-347.

Kumar, M., and Carmichael, G. G. 1998. Antisense RNA: Function and fate of duplex RNA in cells of higher eukaryotes. Microbiol. Mol. Biol.
Rev. 62:1415-1434.

Kunz, C., Schob, H., Stam, M., Kooter, J. M., and Meins, F., Jr. 1996. Developmentally regulated silencing and reactivation of tobacco chitinase transgene expression. Plant J. 10:437-450.

Kwon, C. S., Chung, W. I., and Paek, K. H. 1997. Ribozyme mediated targeting of cucumber mosaic virus RNA 1 and 2 in transgenic tobacco plants. Mol. Cells 7:326-334.

Lindbo, J. A., and Dougherty, W. G. 1992. Untranslatable transcripts of the tobacco etch virus coat protein gene sequence can interfere with tobacco etch virus replication in transgenic plants and protoplasts. Virology 189:725-733.

Lindbo, J. A., Fitzmaurice, W. P., and della-Cioppa, G. 2001. Virus-mediated reprogramming of gene expression in plants. Curr. Opin. Plant Biol. 4:181-185.

Matzke, M. A., and Matzke, A. J. 1995. Homology-dependent gene silencing in transgenic plants: What does it really tell us? Trends Genet. 11:1-3.

Meza, T. J., Kamfjord, D., Hakelien, A. M., Evans, I., Godager, L. H., Mandal, A., Jakobsen, K. S., and Aalen, R. B. 2001. The frequency of silencing in Arabidopsis thaliana varies highly between progeny of siblings and can be influenced by environmental factors. Transgenic Res. 10:53-67.

Montgomery, M. K., Xu, S., and Fire, A. 1998. RNA as a target of doublestranded RNA-mediated genetic interference in Caenorhabditis elegans. Proc. Natl. Acad. Sci. U.S.A. 95:15502-15507.

Morris, T. J., and Dodds, J. A. 1979. Isolation and analysis of doublestranded RNA from virus-infected plant and fungal tissue. Phytopathology 69:854-858.

Murashige, T., and Skoog, F. 1962. A revised medium for rapid growth and bioassays with tobacco tissue cultures. Physiol. Plant. 15:473497.

Napoli, N., Lemieux, C., and Jorgensen, R. 1990. Introduction of chimeric chalcone synthase gene in petunia results in reversible co-suppression of homologous genes in trans. Plant Cell 2:279-289.

Ni, M., Cui, D., Einstein, J., Narasimhulu, S., Vergara, C. E., and SB, G. 1995. Strength and tissue specificity of chimeric promoters derived from octopine and mannopine synthase genes. Plant J. 7:661-676.

Palukaitis, P., Roosinck, M. J., Dietzgen, R. G., and Francki, B. R. I. 1992. Cucumber Mosaic Virus. Adv. Virus Res. 41:281-348.

Pang, S. Z., Jan, F. J., Carney, K., Stout, J., Tricoli, D. M., Quemada, H. D., and Gonsalves, D. 1996. Post-transcriptional transgene silencing and consequent tospovirus resistance in transgenic lettuce are affected by transgene dosage and plant development. Plant J. 9:899-909.

Papaefthimiou, I., Hamilton, A., Denti, M., Baulcombe, D., Tsagris, M., and Tabler, M. 2001. Replicating potato spindle tuber viroid RNA is accompanied by short RNA fragments that are characteristic of post-transcriptional gene silencing. Nucleic Acids Res. 29:2395-2400.

Sambrook, J., Fritsch, E. F., and Maniatis, T. 1989. Molecular Cloning: A Laboratory Manual, 1st ed. Cold Spring Harbor Press, Cold Spring Harbor, NY, U.S.A.

Sijen, T., and Kooter, J. M. 2000. Post-transcriptional gene-silencing: RNAs on the attack or on the defense? Bioessays 22:520-531.

Smith, N. A., Singh, S. P., Wang, M. B., Stoutjesdijk, P. A., Green, A. G., and Waterhouse, P. M. 2000. Total silencing by intron-spliced hairpin RNAs. Nature 407:319-320.

Tabler, M. 1993. Antisense RNA in plants: A tool for analysis and suppression of gene function. Pages 237-258 in: Morphogenesis in Plants. K. A. Roubelakis-Angelakis and K. Tran Thanh Van, eds. Plenum Press, New York.

Vance, V., and Vaucheret, H. 2001. RNA silencing in plants—defense and counterdefense. Science 292:2277-2280.

Vaucheret, H., and Fagard, M,.2001. Transcriptional gene silencing in plants: Targets, inducers and regulators. Trends Genet. 17:29-35.

Vaucheret, H., Palauqui, J. C., Elmayan, T., and Moffatt, B. 1995. Molecular and genetic analysis of nitrite reductase co-suppression in transgenic tobacco plants. Mol. Gen. Genet. 248:311-317.

Voinnet, O., Pinto, Y. M., and Baulcombe, D. C. 1999. Suppression of gene silencing: A general strategy used by diverse DNA and RNA viruses of plants. Proc. Natl. Acad. Sci. U.S.A. 96:14147-14152.

Wang, M. B., Abbot, D. C., and Waterhouse, P. M. 2000. A single copy of a virus-derived transgene encoding hairpin RNA gives immunity to barley yellow dwarf virus. Mol. Plant Pathol. 1:347-356.

Waterhouse, P. M., Wang, M. B., and Lough, T. 2001a. Gene silencing as an adaptive defense against viruses. Nature 411:834-842.

Waterhouse, P. M., Wang, M. B., and Finnegan, E. J. 2001b. Role of short RNAs in gene silencing. Trends Plant Sci. 6:297-301.

Yie, Y., Zhao, F., Zhao, S. Z., Liu, Y. Z., Liu, Y. L., and Tien, P. 1992. High resistance to Cucumber mosaic virus conferred by satellite RNA and coat protein in transgenic commercial tobacco cultivar G-140. Mol. Plant-Microbe Interact. 5:460-465. 